\title{
Anti-tumor Molecular Mechanism of Steroidal Drugs Based on Network Pharmacology
}

\author{
Bian Fuyong \\ Chuxiong Medical College, Chuxiong, China, 675005
}

Keywords: network pharmacology; therapeutic target; Yi nationality medicine; network structure

\begin{abstract}
The purpose was to effectively develop anti-tumor drugs, promote the inheritance and development of ethnic medicine, and study the anti-tumor molecular mechanism of steroid drugs. Method: Based on network pharmacology, the anti-tumor drug constituents of the Yi nationality based on Liangshan Cordyceps were analyzed, the network structure of the Cordyceps system was studied, and the target was predicted. Through multi-channel regulation, the anti-tumor molecular traits of steroid drugs were identified, and comparative experimental tests were carried out on the currently widely used oral anti-tumor drugs. Experimental result: The OB parameters absorbed by the body of the medicinal herbs were detected to understand the relative amount and rate of blood circulation and the molecular mechanism of the drug. Moreover, the current more common drug absorption effect and the absorption effect of the steroid anti-tumor drugs were compared. The test results showed that the absorption performance of the anti-tumor drugs of the Yi nationality with Cordyceps as the main element increased by 30\%-50\% compared with the traditional methods. Result and discussion: By comparing the drug absorption effect and molecular mechanism, it was found that anti-tumor drugs with Cordyceps as the main element can effectively improve the therapeutic effect and reduce the side effects. However, due to the difficulty in clearing the epiphytic bacteria on the surface of Cordyceps, it will have a certain impact on the therapeutic effect of the drug and still needs improvement.
\end{abstract}

\section{Introduction}

With the development of the current medical cause, in order to better develop the medical cause, relevant medical experts have paid more and more attention to the development and promotion of ethnic medicine in order to promote the rapid development of national medicine. Yi medicine has its unique diagnostic and therapeutic technology, unique drug use and good among many ethnic groups. The Yi medicine has better therapeutic effects in many ethnic medicines, especially in the treatment of tumors ${ }^{[1]}$. In order to better cope with the current increasing number of cancer patients, the state has also put forward relevant policies, and actively called on the relevant medical staff of the Yi nationality to extensively explore and collate relevant ancient books, and to understand the important role of $\mathrm{Yi}$ medicine in cancer treatment. In response to the state's call for the development of national medicine, based on network pharmacology, the anti-tumor molecular mechanism of steroid drugs was studied, and the potential value of steroid drugs in treating tumors was analyzed, which exerts the distinctive regional and national characteristics of ethnic medicine and promotes the new development opportunities for national medicine. At present, there are many deficiencies in the analysis and research on the molecular mechanism of steroid drugs in China's medical research system. Therefore, the molecular mechanism of anti-tumor of steroid drugs is analyzed and studied. And the information is input into the pharmacology database and analysis platform of our country to provide reference for the development of Yi medicine.

\section{Material and composition analysis}

\subsection{Yi anti-tumor drug ingredients}

Through a large number of data analysis and literature research, it is found that there are 259 
anti-tumor drugs in Yi medicine, and the average drug contains 1.7×203 molecular components ${ }^{[2]}$. Taking the most representative Liangshan Cordyceps sinensis as the research object, based on pharmacological principles, its chemical composition and molecular structure is collected and analyzed. The molecular structure of the drug is studied using a platform such as Chemical Book, and the molecular structure of the drug is drawn by the benefit software, and the results are uniformly saved. Firstly, the anti-tumor molecular components of the Yi nationality, which are sufficient components of the genus Cordyceps militaris, are analyzed, and the elements such as ergosterol and mannitol are also analyzed ${ }^{[3]}$. Since the chemical constituents of Cordyceps militaris are similar to those of Cordyceps sinensis, the molecular mechanisms of the two are measured and compared, and the following data are obtained.

Table 1 Comparison of anti-tumor molecular chemical composition of steroids

\begin{tabular}{cccc}
\hline $\begin{array}{c}\text { Chemical } \\
\text { composition }\end{array}$ & $\begin{array}{c}\text { Liangshan } \\
\text { Cordyceps \% }\end{array}$ & Comparison of size & $\begin{array}{c}\text { Cordyceps } \\
\text { sinensis \% }\end{array}$ \\
\hline Ergosterol & 1.454 & $<$ & 3.1545 \\
Mannitol & 0.151 & $>$ & 0.052 \\
General saponin & 0.215 & $<$ & 0.151 \\
General flavone & 1.151 & $<$ & 1.456 \\
General nucleoside & 1.541 & $<$ & 1.634
\end{tabular}

Cordyceps is a fungal plant in the plant category, its molecular mechanism components are relatively active, rich in amino acids, pyridone and so on. It has powerful pharmacological effects such as anti-tumor and immune regulation. According to the principle of network pharmacology, Cordyceps has strong effects on tumor treatment, such as enhancing immunity and inhibiting pathogens. This has important correlation with its active ingredients. Therefore, the anti-tumor active components of steroid drugs are detected, and the following data are obtained.

Table 2 Detection of anti-tumor molecular active components of steroid drugs

\begin{tabular}{cccccc}
\hline $\begin{array}{c}\text { Active } \\
\text { ingredients }\end{array}$ & Polysaccharides & Nucleosides & Alditol & Chromones & Nucleosides \\
\hline Threonine & 2.421 & 1.456 & 0.145 & 2.014 & 1.541 \\
Methionine & 2.45 .0 & 1.645 & 1.124 & 2.140 & 0.948 \\
Aspartate & 2.165 & 1.314 & 0.948 & 0.948 & 1.065 \\
Alanine & 1.543 & 1.641 & 0.458 & 1.645 & 1.001 \\
Valine & 1.630 & 1.023 & 1.024 & 1.656 & 0.984 \\
Serine & 1.414 & 1.301 & 0.547 & 0.948 & 1.065 \\
Lysine & 1.326 & 2.012 & 1.541 & 0.996 & 1.215 \\
Isoleucine & 1.355 & 1.450 & 1.035 & 0.845 & 2.018 \\
Leucine & 0.615 & 1.614 & 1.321 & 1.054 & 2.154 \\
Phenylalanine & 0.511 & 1.510 & 1.549 & 1.068 & 1.664 \\
Tryptophan & 0.946 & 1.321 & 1.044 & 1.846 & 2.064 \\
L-cysteic acid & 0.868 & 1.510 & 0.487 & 0.948 & 1.064 \\
Arginine & 1.464 & 0.975 & 1.242 & 0.642 & 1.245 \\
Histidine & 0.954 & 1.064 & 0.324 & 0.346 & 1.349 \\
Tyrosine & 1.645 & 2.645 & 2.564 & 1.212 & 0.942 \\
\hline
\end{tabular}

\subsection{Molecular network screening and target prediction based on pharmacology}

Traditional anti-tumor drugs are mostly oral preparations, which need to be combined with target organs and target tissues through absorption and metabolism of the human body, thereby slowly exerting the anti-tumor drugs. Liangshan Cordyceps is clinically an oral drug. Therefore, in the course of research, it is necessary to combine the professional medical molecular research software to calculate and analyze the molecular mechanism of the compound, and to screen out the potential active components in the molecular components of the drug. According to the active component of 
the drug, target matching is performed. In order to better predict the anti-tumor molecular mechanism of the drug, molecular mechanism correction and transformation operations are performed on the first 12 targets which are well interfaced with the pharmaceutically active component. Therefore, the relevant protein targets can be more accurately obtained, and the target protein data can be imported, thereby effectively analyzing the type of the anti-tumor drug, and timely eliminating and classifying the target without the disease information.

\subsection{Molecular network structure and trait identification of anti-tumor drugs in Yi nationality}

Due to the richness and diversity of drugs in China, most medical workers currently only conduct in-depth research and accurate identification of a certain species, and there is still a great lack of research on anti-tumor drugs of the Yi nationality. Therefore, based on the network pharmacology, the DNA values of the drug molecules were identified, and the biomolecules of Cordyceps militaris were accurately identified. Based on the timely investigation and identification of drug molecules in combination with network pharmacology, the current identification method is used to analyze and study the molecular traits and mechanisms of Cordyceps militaris. By comprehensively observing and studying the experimental samples in combination with the antitumor molecular characteristics of the medicinal materials, the molecular traits and microscopic identification methods were optimized. It can effectively supplement the characteristics of the worm's ring and segmentation of Cordyceps. In the microscopic study of molecular characteristics of steroid drugs, the internal hyphae and sub-slices and powder characteristics of Cordyceps were studied.

\section{Experimental methods and results analysis}

\subsection{Experimental equipment}

In order to better study the anti-tumor molecular mechanism of the Yishan Cordyceps militaris, high-precision solid microscope, detection platform digital imaging system, medical image capture software, transmitted light microscope and image processing software are needed in the experiment.

During the experiment, $75 \mathrm{~g}$ of chloral hydrate, $12 \mathrm{ml}$ of water, and $60 \%$ of dilute glycerin and $30 \mathrm{ml}$ of glycerol, and a small piece of camphor were selected to obtain $15 \mathrm{ml}$ of chloral hydratedissolved test oil. In addition to the Liangshan Cordyceps sinensis, some solutions such as ethanol, xylene and iodine are needed during the experiment.

\subsection{Test method and result analysis}

The surface of the sample of the Cordyceps militaris was infiltrated with water, and the residual membrane and impurities were removed, and the cordyceps and the larvae were cut into small pieces of $10 \mathrm{~mm}$. Moreover, the appropriate amount of the sample is removed, embedded in paraffin, stained, and then inoculated into the prepared chloral hydrate dissolution test oil, and placed on a glass slide to prepare a temporary loading. The molecular mechanism of the drug is observed and recorded by the imaging system of the high-power microscope detection platform, and the $\mathrm{OB}$ parameters absorbed by the body of the medicinal materials are detected according to the network pharmacology method. Therefore, the relative amount and rate of blood circulation in tumor patients can be understood, and the training set of metabolic cytochrome and transport glycoprotein information can be integrated, and the microscopic characteristics and elemental absorption effects of the drug can be analyzed. The data are recorded as follows.

Table 3 Test results of absorption factors of medicinal materials of the Yi nationality

\begin{tabular}{cccccccc}
\hline Name & $\mathrm{Zn}$ & $\mathrm{Fe}$ & $\mathrm{Ca}$ & $\mathrm{Co}$ & $\mathrm{Mn}$ & $\mathrm{Mg}$ & $\mathrm{Pb}$ \\
\hline Cordyceps & 103.14 & 56.48 & 76.15 & 15.15 & 106.48 & 121.42 & 106.48 \\
$\begin{array}{c}\text { Cordyceps } \\
\text { sample }\end{array}$ & 100.12 & 58.45 & 76.15 & 14.45 & 106.84 & 125.41 & 106.84 \\
$\begin{array}{c}\text { Cordyceps } \\
\text { powder }\end{array}$ & 98.15 & 55.04 & 79.12 & 16.42 & 106.51 & 126.45 & 105.94 \\
\hline
\end{tabular}




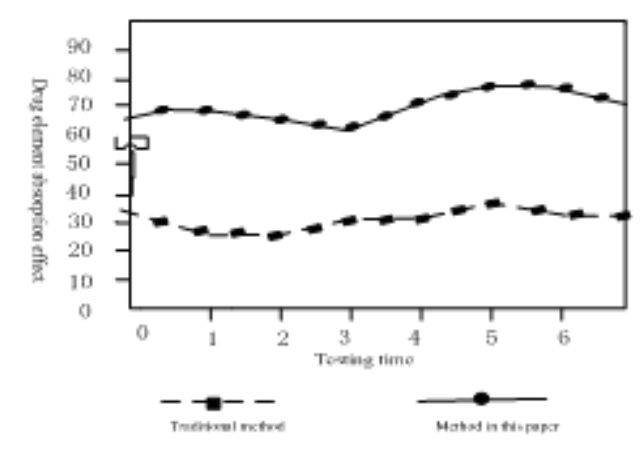

Figure 1 Experimental comparison test results

Drug absorption is an important test factor for the effectiveness of the molecular mechanism of anticancer drugs. Compared with the molecular absorption effect of traditional oral anticancer solutions, the results can be obtained.

\subsection{Discussion of experimental results}

Through the observation and research on the samples of Cordyceps militaris, it was found that for the traditional anti-tumor oral liquid, the elemental absorption of the medicinal materials mainly based on Cordyceps is relatively good. However, it was found that the epiphytic bacteria on the surface of Cordyceps militaris were not easy to be removed. After the sample was air-dried, the body wall folds and depressions were easy to occur, which caused the molecules to be difficult to observe. It was easy to affect the drug effect and performance, and further research and optimization were needed. In addition, in the process of high-intensity ratio microscopy to detect the molecular mechanism of drugs, it is found that there are lateral bifurcations in the molecular mechanism of Cordyceps. Clinical studies have confirmed that this structure is beneficial for the inhibition of tumor cells. Therefore, it can be confirmed that the anti-drugs with Cordyceps as the main component have better research and development value in anti-tumor.

\section{Conclusion}

Malignant tumors are a serious threat to people's lives and health and a great obstacle to the development of our society. China is a multi-ethnic country, and national medicine is a valuable asset in China's medical system. Therefore, combined with the molecular mechanism of the medicinal materials of the Yi nationality, the treatment and anti-tumor methods of the tumor were analyzed and studied. It was confirmed by experiments that the drug based on Liangshan Cordyceps has good curative effect on anti-tumor and has great potential in treating tumor.

\section{Acknowledgement}

Yunnan provincial department of education scientific research fund project(No. 2018JS633)

\section{References}

[1] Zhang Xinzhuang, Xiao Wei, Xu Xiaojie, et al. Molecular mechanism of action of Guizhi Fuling Capsules in the treatment of dysmenorrhea, uterine fibroids and pelvic inflammatory disease based on network pharmacology[J]. Chinese Traditional and Herbal Drugs, 2016, 47(1): 81-94.

[2] Sun Limin, Liu Lifang, Zhu Huaxu, et al. Mechanism of Huanglian Jiedu Decoction in the treatment of Alzheimer's disease based on network pharmacology [J]. Journal of Pharmaceutical Sciences, 2017,36(8): 1268-1275.

[3] Luo Xuan. Study on the basis and molecular mechanism of anti-influenza substances of honeysuckle, astragalus and forsythia based on network pharmacology [D]. Shaanxi University of 
Traditional Chinese Medicine, 2016,16(8):26-35.

[4] Zhang Jianyong, Wang Lan, Liang Rixin, et al. Analysis of the mechanism of antiatherosclerosis in the distribution of Danshen Hawthorn group based on network pharmacology[J]. Chinese Journal of Traditional Chinese Medicine, 2016, 41(23): 4408-4415.

[5] Wang Yanping, Pan Liwei, Wei Zheng, et al. Study on the Mechanism of Anti-Rheumatoid Arthritis of Yaofang Medicine Based on Network Pharmacology [J]. Volkswagen Science, 2017, 19(4): 68-70. 